\title{
Panton Valentine Leucocidin (PVL) positive staphylococcal infection: an emerging infection across the world
}

\author{
Harris Pathirage ${ }^{1}$ \\ Sri Lanka Journal of Child Health, 2008; 37: 109-111
}

(Key words: Panton Valentine Leucocidin (PVL) positive strains, Staphylococcus aureus)

A new pattern of disease is emerging worldwide due to Panton Valentine Leucocidin (PVL) positive strains of Staphylococcus aureus. PVL is a toxin secreted by certain strains of Staphylococcus aureus which can be detected in both methicillin sensitive and resistant strains of staphylococci ${ }^{1}$. PVL positive $S$. aureus is normally associated with necrotizing pyogenic cutaneous skin lesions, cellulitis and tissue necrosis. It can also cause more severe infections such as septic arthritis, bacteraemia, purpura fulminans and community acquired necrotising pneumonia $^{2}$.

PVL toxin was first described by van de Velde in 1894 due to its ability to lyse leucocytes. It was named after Panton and Valentine when they associated it with soft tissue infections in $1932^{3}$. Infections caused by PVL positive strains have been documented since 1930 but are much more prevalent now. Genetic analysis shows that PVL community acquired methicillin resistant staphylococcus aureus (MRSA) has emerged several times in different continents, rather than being the worldwide spread of a single clone ${ }^{4}$.

Less than 2\% of Staphylococcus aureus are PVL positive in the United Kingdom and it includes both methicillin sensitive and resistant strains ${ }^{5}$. PVL positive strains are usually community acquired and generally affect healthy children and young adults. However, hospital acquired MRSA strains generally do not produce PVL and are more commonly associated with wound infections and septicaemia in severely ill patients ${ }^{6}$.

Nearly all strains of Staphylococcus aureus secrete exotoxins, which constitute an essential component of virulent mechanisms. For example, haemolysins, lipases, proteases, hyaluronidases and collagenases convert host tissues into nutrients required for bacterial growth. Some strains produce additional

${ }^{1}$ Consultant Paediatrician, Special Care Baby Unit, Kethumathi Maternal Hospital, Panadura. exoproteins that may be responsible for particular clinical manifestations. PVL is one of them. Other exoproteins are staphylococcal enterotoxins, toxic shock syndrome toxin-1 and exfoliative toxins ${ }^{7}$.

PVL is a bicomponent cytotoxin encoded by two contiguous and cotranscribed genes of staphylococcus genome carried on a bacteriophage ${ }^{8}$. The 2 genes are responsible for production of 2 secretory proteins which act together as subunits assembling in the membrane of host defence cells particularly white blood cells, monocytes and macrophages 9 . The subunits fit together and form a ring with a central pore through which cell contents leak and which themselves act as superantigens ${ }^{10}$. The PVL toxin, produced by less than $5 \%$ of the $S$. aureus isolates, causes leucocyte destruction and tissue necrosis which result in necrotic skin lesions and severe necrotizing pneumonia.

PVL strains of $S$. aureus affect mainly healthy, immunocompetent children and young adults. Asymptomatic carriage has also been reported. There are two main disease entities clinically. The common presentation is purulent skin infection with cellulitis, abscesses, boils and carbuncles. The rare but severe life threatening form includes invasive infections such as septic arthritis, bacteraemia and necrotising pneumonia. PVL pneumonia presents without skin lesions but often has a preceding influenza like illness. It causes a rapidly progressive, haemorrhagic, necrotizing, community acquired pneumonia, followed by hypotension and shock within 2-3 days without proper management ${ }^{11}$. A high degree of suspicion is needed in healthy children and young adults, presenting with a similar clinical picture to prevent high mortality, reported to be as high as $70 \%$ in one series ${ }^{12}$.

Polymerase chain reaction (PCR) test for PVL virulence genes is the hallmark of diagnosis ${ }^{13}$. Supportive tests include leucopenia caused by the destruction of leucocytes by PVL toxin and very high $\mathrm{C}$-reactive proteins. Chest $\mathrm{X}$-ray shows multilobular 
infiltrates accompanied by effusion and cavitation. Gram stain on sputum film demonstrates sheets of gram positive staphylococci. Swabs taken for culture from the skin lesions are positive for $S$. aureus. Further discrimination can be done to identify the methicillin resistant and sensitive strains.

All suspected patients should be managed in an intensive care unit (ICU). An aggressive intravenous antibiotic policy should be adopted. Several publications have reported the value of combination therapy. Combinations, including flucloxacillin, vancomycin, clindamycin, linezolid, rifampicin and cotrimoxazole in high doses have been used ${ }^{14}$. Flucloxacillin may be used for its bactericidal action in combination with linezolid or rifampicin. Linezolid can be used to cover MRSA pending antibiotic susceptibility results. Clindamycin and linezolid have an added advantage of suppressing toxin production. Adjunctive treatment with intravenous immunoglobulin (IVIG) $2 \mathrm{~g} / \mathrm{kg}$ could be considered in very ill PVL positive $S$. aureus infections. It neutralizes exotoxins and superantigens. Activated protein $\mathrm{C}$ also has been used in some centres but should be avoided in active pulmonary haemorrhage.

Staphylococcus aureus colonizes approximately 30\% of general population and up to $50 \%$ of intravenous drug users, diabetics and health care workers ${ }^{15}$. The organism is transmitted between persons by close contact. Therefore, preventive measures aim at decolonizing carriers by means of nasal mupirocin, chlorhexidine showers and alcoholic hand rubs for those at risk of infections or transmission ${ }^{16}$. Screening (nose, axilla, perineum, throat, skin lesions) of close contacts for carriage of PVL positive $S$. aureus would help to prevent spread of the infection ${ }^{17}$. For the clinical staff, infection control measures like closed tracheal suction, wearing surgical masks during intubation and physiotherapy, should be adopted.

Although PVL toxin, secreted by Staphylococcus aureus strains have got a reported history extending for more than one hundred years, it has not been a talking point until recently. Advances of technology, facilities for detection of PVL positive $S$. aureus and growing interest in reporting and scientific research have contributed to highlight this condition in the recent past. Furthermore, the fatal outcome of majority of undiagnosed and poorly managed cases needs to be considered.
Three salient features of this infection viz. community acquired nature, affected group being otherwise healthy, immunocompetent children and young adults, and the rapid course of the infection leading to haemorrhage, shock and death, distinguish it from traditional $S$. aureus infection.

Easily adoptable preventive measures are available both in the community and hospitals to prevent further spread of the PVL positive $S$. aureus strains. More attention should be paid to this condition in the developing world as it may be under-diagnosed due to lack of facilities for genetic analysis of the PVL positive strain or diagnosis may be extrapolated on other conditions of similar clinical spectrum. Therefore it is the duty of both clinicians and health administrators to be vigilant about it and take necessary measures to avoid untimely deaths of healthy children and young adults from this preventable condition.

\section{References}

1. Davis SL, Perri MB, Donabedian SM, Manierski IC, et al. Epidemiology and outcomes of Community Associated Methicillin Resistant Staphylococcus aureus infection. $J$ Clin Microbiol 2007; 45 (6): 1705-11.

2. Szmiegielski S, Prevost G, Monteil, et al. Leukocidal toxins of staphylococci Zentralbl Bakteriol 1999; 289:185-201.

3. Panton PN, Valentine FCO, Came MB. Staphylococcal Toxin. The Lancet 1932; 1: 5068.

4. MRSA Toxin Acquitted: Study Clears Suspected Key to Severe Bacterial Illness, NIH News Release, Nov 6, 2006. Available from: http://www3.niaid.nih.gov/news/newsreleases/20 06/staphtoxin.htm

5. Holmes A, Ganner M, McGuane S, et al. Staphylococcus aureus isolates carrying Panton Valentine leucocidin genesin England and Wales: frequency, characterization and association with clinical disease. $J$ Clin Microbiol. 2005; 43 (5): 2384-90.

6. Gillet $\mathrm{Y}$, Issartel $\mathrm{B}$, Vanhems $\mathrm{P}$, et al. Association between Staphylococcus aureus strains carrying gene for Panton Valentine leukocidin and highly lethal necrotizing pneumonia in young immunocompetent patients. Lancet 2002; 359 (9308): 753-9. 
7. Dinges MM, Orwin PM, Schlievert PM. Exotoxins of Staphylococcus aureus. Clin Microbiol Rev 2000;13: 16-34.

8. Lina G, Piamont Y, Godail, Gamot F, et al. Involvement of Panton Valentine leukocidin producing Staphylococcus aureus in primary skin infections and pneumonia. Clin Infect Dis 1999; 29:1128-32.

9. Melles DC, van Leeuwen WB, Boelens HAM, Peeters JK, et al. Panton Valentine leukocidin genes in Staphylococcus aureus. Emerg Infect Dis 2006 July. Available from:

http://www.cdc.gov/NCIDOD/EID/vol12no07/0 5-0865.htm

10. Bradley SF. Staphylococcus aureus Pneumonia: Emergence of MRSA in the community. Semin Respir Crit Care Med 2005; 26 (6): 643-9.

11. Lim WS, Van der Eerden MM, Laing R, et al. Defining community acquired pneumonia severity on presentation to hospital and international derivation and validation study. Thorax 2003; 58 (5): 377-82.

12. Dufour P, Gillet Y, Bes M, Lina G, et al. Community acquired methicillin resistant Staphylococcus aureus infection in France: emergence of a single clone that produces Panton
Valentine leukocidin. Clin Infect Dis 2002; 35: 819-24.

13. McClure JA, Conly JM, Lau V, et al. Novel multiplex PCR assay for detection of the Staphylococcal virulence marker Panton Valentine leukocidin genes and simultaneous discrimination of methicillin susceptible from resistant staphylococci. J. Clin Microbiol 2006; 44 (3): 1141-4.

14. Steven DL, Ma Y, Salmi DB, et al. Impact of Antibiotics on Expression of Virulence Associated Exotoxin Genes in Methicillin Sensitive and Methicillin Resistant Staphylococcus aureus. J Infect Dis 2007; 195 (2): 202-11.

15. Archer GL. Staphylococcus aureus: a well armed pathogen. Clin Infect Dis 1998; 26: 117981.

16. Boyce JM. MRSA patients: proven methods to treat colonization and infection. J Hosp Infect 2001; 48: 9-14.

17. Coia JE, Duckworth GJ, Edwards DI, et al. Guidelines for the control and prevention of methicillin resistant Staphylococcus aureus in healthcare facilities. J Hosp Infect 2006; 63 Suppl: S1-44. 